\title{
Argumentación científica escolar: modelo para la defensa del medio ambiente
}

\author{
Adriana Valenzuela-González ${ }^{1}$ \\ Laura Zuñiga-González²
}

Fecha de recepción: 21 de marzo de 2020

Fecha de aprobación: 9 de mayo de 2020

\section{Resumen}

Esta propuesta de investigación está orientada al planteamiento, validación, implementación y evaluación de un modelo de argumentación científica que promueva el reconocimiento, en estudiantes de básica secundaria de la Institución Educativa Ciudad Verde del municipio de Soacha, de las energías renovables como una estrategia de reducción de los problemas ambientales. Se plantea una metodología de tipo mixto con la intención de aprovechar sus componentes tanto cualitativos como cuantitativos. Como resultado, se describen de forma general los elementos teóricos que permitirán la construcción de la propuesta de modelo argumentativo. Se concluye indicando la importancia de desarrollar procesos argumentativos que contribuyan a la superación de la crisis climática global. Esto a través de acciones educativas que fortalezcan en los ciudadanos el desarrollo del pensamiento crítico para que, de una forma participativa, puedan contribuir en la mitigación de los problemas ambientales actuales.

\footnotetext{
${ }^{1}$ Ph. D. (c) Universidad Distrital "Francisco José de Caldas" (Bogotá-Distrito Capital, Colombia). ORCID: 00000002-4241-1823

2 Ph. D. (c) Universidad Distrital "Francisco José de Caldas" (Bogotá-Distrito Capital, Colombia). ORCID: 0000$\underline{0001-6002-8345}$
} 
Palabras clave: argumentación científica; educación en energías renovables; problemáticas ambientales.

\section{School Scientific Argumentation: Model for the Defense of the Environment}

\section{Abstract}

This research proposal is aimed to the approach, validation, implementation and evaluation of a scientific argumentation model that promotes the recognition of renewable energies as a strategy to reduce environmental problems in elementary school students of the Ciudad Verde Educational Institution, at the municipality of Soacha. A mixed type methodology is presented, with the intention of taking advantage of its qualitative and quantitative components. As a result, the theoretical elements that will allow the construction of the argumentative model proposal are generally described. The conclusion indicates the importance of developing argumentative processes that contribute to overcoming the global climate crisis. This, through educational actions that strengthen the development of critical thinking, so that citizens can contribute in a participatory way to the mitigation of current environmental problems.

Keywords: environmental problems; renewable energy education; scientific argumentation.

\section{Para citar este artículo:}

Valenzuela-González, A., \& Zuñiga-González, L. (2020). Argumentación científica escolar: modelo para la defensa del medio ambiente. Pensamiento y Acción, 28, 35-46.

Esta obra está bajo licencia internacional Creative Commons Reconocimiento 4.0 


\section{Introducción}

Este proyecto de investigación busca generar responsabilidades ambientales a través del desarrollo de procesos argumentativos en los estudiantes del municipio de Soacha - Cundinamarca (Sauvé, Bernard \& Sloan, 2016). Esto hace necesario plantearse el desarrollo e implementación de un modelo argumentativo de educación en ciencias que se base fundamentalmente en el conocimiento de las energías renovables como estrategia de reducción de los problemas ambientales. Se espera que este proceso educativo permita orientar al cambio de valores, concepciones y actitudes de la humanidad con el medio ambiente (Porter \& Van Der-Linde, 1995; Cutter et al., 2008; Huxster, Uribe-Zarain, \& Kempton, 2015; Mirandola \& Lorenzini, 2016).

Para el cumplimiento de este propósito, se estructuran dos categorías que se establecen como ejes en la búsqueda documental y construcción teórica: argumentación científica escolar y educación en energías renovables; aunadas a la categoría de pensamiento crítico que se desarrollará de manera transversal. Esta última tendrá relevancia en el desarrollo de los procesos argumentativos, dado que, investigar sobre las prácticas comunicativas y cognitivas de la vida cotidiana en diversas comunidades, permite la comprensión del desarrollo cognitivo en contextos familiares e institucionales (Rogoff \& Chavajay, 2004). De igual forma, de acuerdo con Castro \& Gallego (2015), fortalecerá la creación de una cultura energética en el arraigo de una ética personal de consumir lo necesario, no contaminar el ambiente, participar en la creación de riquezas para la comunidad y, con su ejemplo, transmitir experiencias positivas, además de buscar alternativas tecnológicas que permitan resarcir los daños causados a los ecosistemas.

\section{Argumentación Científica Escolar}

Diversas investigaciones en el campo de la argumentación científica han mostrado cómo esta categoría conceptual puede ser analizada desde múltiples dimensiones que relacionan el desarrollo de la habilidad cognitivo-lingüística con el rendimiento académico, el aprendizaje, las habilidades de discusión, las creencias epistemológicas, la comprensión conceptual, la naturaleza de las ciencias, las 
habilidades de pensamiento científico, o la calidad del argumento, entre otras (Bogar, 2019; Gallego, Valenzuela \& Ballesteros, 2019).

De igual forma, el amplio espectro de investigaciones que se encuentran disponibles sobre argumentación científica escolar y la propuesta de modelos argumentativos como Toulmin (1958), Giere (1991), Schwarz, Neuman \& Gil Ve İlya (2003), Zohar \& Nemet (2002), Kelly \& Takao (2002), Lawson (2003), Sandoval (2003), McNeill et al. (2006), entre otros citados por Aktamiş \& Hiğde (2015), dan cuenta de la importancia de formular modelos argumentativos que se ajusten a entornos educativos concretos y que puedan dar respuesta a las necesidades educativas puntuales. Para el objetivo de esta propuesta de investigación, la necesidad es identificar las problemáticas ambientales y promover el conocimiento de las energías renovables en el municipio de Soacha.

La argumentación se considera una habilidad esencial para la formación científica ciudadana (Felton, Garcia-Mila, Villarroel \& Gilabert, 2015; Nielsen, 2012; Duschl, \& Osborne 2002; Jiménez-Aleixandre, Bugallo \& Duschl, 2000; Newton, Driver \& Osborne, 1999), debido a que los procesos argumentativos constituyen una práctica esencial en la construcción del conocimiento científico, a través de la crítica, la replicación y la evaluación (Dawson \& Carson, 2017).

En este sentido, el modelo de argumentación científica escolar que se plantea procura revisar y fortalecer los argumentos que se encuentran presentes en los diálogos, socialmente necesarios, que los estudiantes sostienen en la clase de ciencias naturales acerca del reconocimiento de las problemáticas ambientales. Destaca también la forma en la que el conocimiento de las energías renovables procurará establecer posturas críticas frente a esta problemática tan necesaria en la actualidad (Ocetkiewicz, Tomaszewska \& Mróz, 2017; Martín-Gámez \& Erduran, 2018).

El análisis que se espera realizar comprende los argumentos como ideas que se encuentran insertas en diálogos producidos en contextos de clase de ciencias naturales y responden a objetivos concretos. Walton (1998) citado por Kolstø y Ratcliffe (2007) presenta una clasificación de diálogos que intenta cubrir todo tipo de interacciones argumentativas, así mismo, define el diálogo como "un marco 
normativo en el que existe un intercambio de argumentos entre los socios que razonan juntos y, a su vez, toman la secuencia dirigida a un objetivo colectivo" (p. 30). Los autores afirman la existencia de cinco tipos diferentes de diálogos caracterizados, entre otros atributos, por diferentes objetivos: diálogo de persuasión, diálogo de búsqueda de información, diálogo de negociación, diálogo de investigación y diálogo erístico.

Para cumplir con los objetivos de la propuesta de investigación, se tendrán en cuenta dos tipos de diálogo que son importantes como representaciones de prácticas científicas. La discusión crítica vista como un tipo de diálogo de persuasión y la investigación científica "como un tipo de diálogo de investigación de interés social en nuestro contexto, debido a su posible relevancia para describir los discursos científicos" (Kolstø y Ratcliffe, 2007).

Lo anterior resalta la necesidad de desarrollar los procesos argumentativos de los estudiantes de una institución educativa secundaria del municipio de Soacha de tal manera que puedan construir y analizar argumentos relacionados con las implicaciones y aplicaciones sociales de la ciencia. Esto involucra la capacidad de comprometerse con las afirmaciones de las fronteras de la ciencia comprendidas en temas socio-científicos controvertidos (Kolstø \& Ratcliffe, 2007), brinda la oportunidad de analizar las situaciones científicas que ocurren alrededor, y provee las herramientas conceptuales, cognitivas y lingüísticas para dar a conocer un punto de vista y el aporte con alternativas de solución efectivas. Como resultado, el estudiante será un agente activo ante las diversas problemáticas ambientales a las que se enfrenta (Sánchez \& Torres, 2016).

\section{Educación en energías renovables}

Actualmente ha surgido la necesidad de incrementar la producción energética que supla las demandas mundiales, cuyos factores de crecimiento están determinados por razones diversas, como el aumento de la población, el rápido desarrollo y la urbanización, (Guven \& Sulun 2017; Ballesteros \& Gallego, 2019a). Sin embargo, en el proceso de consecución de los recursos energéticos, la depredación y el uso de los recursos de la naturaleza ha sido inclemente, hasta el punto de llegar casi a 
la extinción de elementos naturales, así como al recrudecimiento de fenómenos naturales como el calentamiento global, entre otros, que afectan a la población a nivel mundial. En este sentido, es necesario desarrollar procesos de sensibilización ambiental en la producción de energía, a través de la educación energética que pueda darse desde los primeros niveles de escolarización, y que permita la generación de cambios en la forma en que se comprende el uso y la transformación de la energía (Ballesteros \& Gallego 2019b).

El proceso de educación en energías renovables se constituye en un papel mediador que considera las necesidades sociales y ambientales de los contextos en los que puedan establecerse sus propuestas. Sus propósitos principales son lograr una aceptación social de las energías Renovables ER, permitir que los estudiantes se conviertan en consumidores de energía responsables, y que éstos desarrollen actitudes proambientales, (Zyadin et al., 2012). Diferentes investigaciones (DeWaters \& Powers, 2011; Lee et al., 2015), han reconocido la necesidad de que la educación en energías renovables pueda darse a niños y adolescentes de tal forma que influya en el comportamiento del consumo de energía en la edad adulta. En este sentido, "educar a las nuevas generaciones para que sepan leer y escribir sobre energía es esencial para conservar la energía y garantizar la sostenibilidad" (Lee, Lee, Wu \& Pan, 2019)

Kandpal y Garg (1999), plantearon los siguientes objetivos de la educación en energías renovables, postulados que aún conservan vigencia y que se hace necesario incluir en los programas de educación en energía renovable, tanto en la escuela, como fuera de ella:

(i) Desarrollar una conciencia entre los estudiantes sobre la naturaleza y la causa de la crisis energética actual.

(ii) Concienciar a los estudiantes sobre diversos tipos de fuentes de energía no renovables y renovables, el potencial de sus recursos y las tecnologías existentes para aprovecharlos.

(iii) Proporcionar a los estudiantes las habilidades necesarias para aprovechar diversas fuentes de energía. 
(iv) Hacer que los estudiantes comprendan las consecuencias de varias medidas de políticas relacionadas con la energía. (p. 74)

Los objetivos anteriores demuestran que el papel de la ciudadanía es fundamental en el cambio de perspectiva en cuanto al uso de energías, puesto que el proceso de concienciación, desarrollo de valores y actitudes, y la comprensión en general de las consecuencias del uso de energías, solo puede lograrse cuando existe participación y una buena comunicación que propicie la toma de decisiones en la comunidad (Liarakou, Gavrilakis \& Flouri, 2009; Rozo-Rincón, Sánchez-Molina \& Gelves-Díaz 2014). Asimismo, se necesitan actitudes sociales que permitan divulgar los beneficios de un cambio de modelo energético.

\section{Referente metodológico}

Se plantea una metodología de tipo mixto, con la intención de aprovechar sus componentes tanto cualitativos como cuantitativos (Creswell, 2014). La población a la que está dirigida comprende estudiantes de grado noveno (14-16 años) de básica secundaria de la Institución Educativa Ciudad Verde, en el municipio de Soacha. El diseño metodológico está estructurado en seis fases como se indica en la Figura 1.

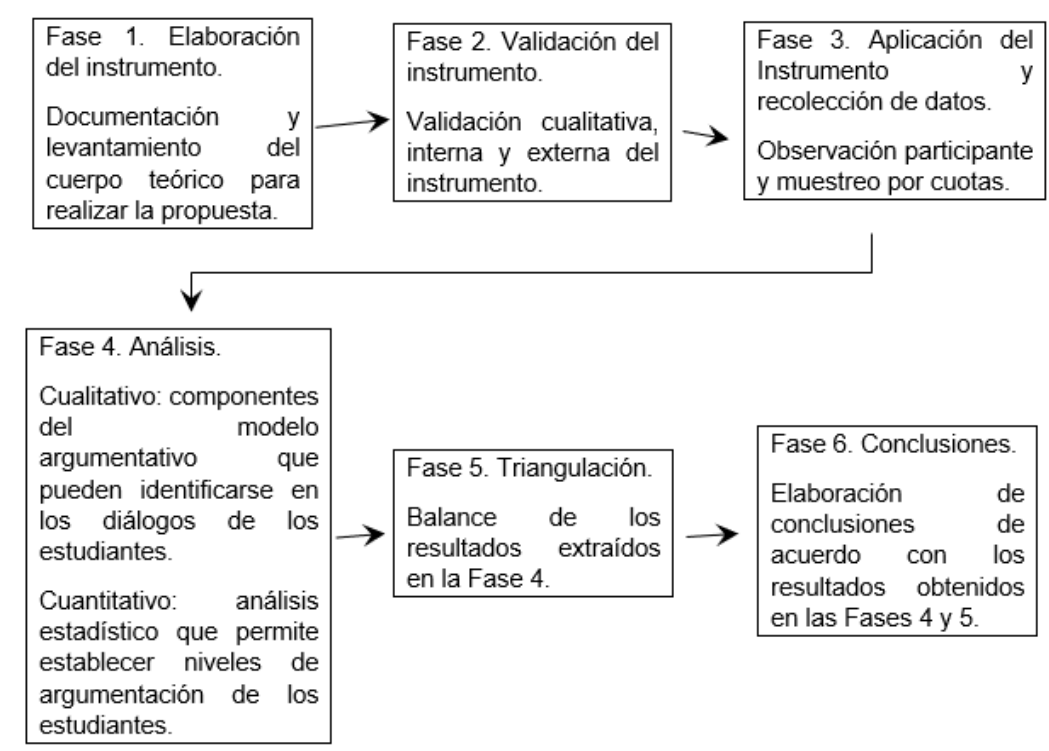

Fig. 1. Fases de la investigación. 


\section{Resultados y discusión}

Se pretende implementar de forma exitosa un modelo de argumentación científica que fortalezca en los estudiantes de educación secundaria la competencia argumentativa con el objetivo de que, a través del análisis de los diálogos que se presentan en la clase de ciencias y la ejecución del modelo propuesto, se establezcan premisas sólidas respecto a las energías renovables como alternativa eficaz en la reducción de las problemáticas ambientales del municipio de Soacha.

Como resultado parcial, se considera la construcción teórica partiendo del rastreo y análisis documental de las categorías conceptuales, lo que permitirá la estructuración del modelo a través del cual se podrá realizar una clasificación de los procesos argumentativos de los estudiantes. Del mismo modo, le permitirá al docente de ciencias obtener un diagnóstico de esta competencia en sus estudiantes y convertir la clase en un espacio pedagógico para el desarrollo del pensamiento crítico, cuyo propósito es generar un "impacto social de la ciencia" (Pinch, 2015).

Acudir a los procesos argumentativos en la educación científica supone comprender la Naturaleza del Conocimiento Científico y sus objetivos para la enseñanza científica y escolar, así como para la enseñanza de las ciencias para la ciudadanía. Esto implica que los estudiantes no solo deben comprender los conceptos bajo los cuales se desarrolló la ciencia, sino que deben ser capaces de aplicarlos en su vida diaria y, más aun, en el momento histórico que atravesamos actualmente, donde el cuidado de medio ambiente y las fuentes energéticas son el principal camino para evitar que se extinga la raza humana. En este sentido, acudir a los procesos argumentativos resulta imperativo a la hora de co-construir una visión más adecuada de la naturaleza de la ciencia y desarrollar perspectivas críticas en los estudiantes que les permitan procesos de enseñanza-aprendizaje alejados de los modelos habituales de trasmisión- recepción.

\section{Referencias}

Aktamiş, H., \& Hiğde, E. (2015). Assessment of Argumentation Models Used in Science Education. Mehmet Akif Ersoy Üniversitesi Eğitim Fakültesi Dergisi, 1 (35), 136-172 
Ballesteros-Ballesteros, V., \& Gallego-Torres, A. P. (2019a). La educación en energías renovables desde las controversias socio-científicas en la educación en ciencias. Revista Científica, 2(35), 192-200. https://doi.org/10.14483/23448350.14869

Ballesteros-Ballesteros, V., \& Gallego-Torres, A. P. (2019b). Modelo de educación en energías renovables desde el compromiso público y la actitud energética. Revista Facultad de Ingeniería, 28(52), 27-42. https://doi.org/10.19053/01211129.v28.n52.2019.9652

Bogar, Y. (2019). Revisión de Estudios Nacionales e Internacionales sobre Argumentación Científica en Educación. Sınırsız Eğitim ve Araştırma Dergisi, 4 (1), 90-120. https://doi.org/10.29250/sead.494930

Castro-Montaña, J. E., \& Gallego-Torres, A. P. (2015). La educación energética una prioridad para $\begin{array}{lllll}\text { el milenio. } & \text { Revista } & \text { científica, } & 1 & (21),\end{array}$ https://doi.org/10.14483/udistrital.jour.RC.2015.21.a11

Creswell, J. (2014). Una introducción concisa a la investigación de métodos mixtos. Publicaciones sabias.

Cutter, L., Barnes, L., Berry, M., Burton, C., Evans, E., Tate, E., \& Webb, J. (2008). A place-based model for understanding community resilience to natural disasters. Global environmental change, 18 (4), 598-606. https://doi.org/10.1016/j.gloenvcha.2008.07.013

Dawson, V., \& Carson, K. (2017). Using climate change scenarios to assess high school students' argumentation skills. Research in Science \& Technological Education, 35 (1), 1-16. https://doi.org/10.1080/02635143.2016.1174932

DeWaters, J. E., \& Powers, S. E. (2011). Energy literacy of secondary students in New York State (USA): A measure of knowledge, affect, and behavior. Energy policy, 39 (3), 1699-1710. https://doi.org/10.1016/i.enpol.2010.12.049

Duschl, R. A., \& Osborne, J. (2002). Supporting and promoting argumentation discourse in science education. Studies in Science Education, $38 \quad$ (1), $39-72$. https://doi.org/10.1080/03057260208560187

Felton, M., Garcia-Mila, M., Villarroel, C., \& Gilabert, S. (2015). Arguing collaboratively: Argumentative discourse types and their potential for knowledge building. British Journal of Educational Psychology, 85 (3), 372-386. https://doi.org/10.1111/bjep.12078

Gallego-Badillo, R., Pérez- Miranda, R., Gallego-Torres, A. P., \& Guzmán, D. B. (2020). Research on Colombian Science Teachers' Education: A Review. Science Education Research in Latin America, 2020, 207-226. https://doi.org/10.1163/9789004409088 009

Gallego-Torres, P., Valenzuela González, A, \& Ballesteros-Ballesteros, V. (2019). Argumentación científica escolar: una propuesta para la defensa del medio ambiente. Universitas Tarraconensis. Revista de Ciències de l'Educació, 2019, 145-153. https://doi.org/10.17345/ute.2019.2.2656 
Argumentación científica escolar: modelo para la defensa del medio ambiente

Guven, G., \& Sulun, Y. (2017). Pre-service teachers' knowledge and awareness about renewable energy. Renewable and Sustainable Energy Reviews, 80, 663-668. https://doi.org/10.1016/i.rser.2017.05.286

Huxster, J. K., Uribe-Zarain, X., \& Kempton, W. (2015). Undergraduate understanding of climate change: The influences of college major and environmental group membership on survey knowledge scores. Journal of Environmental Education, 46 (3), 149-165. https://doi.org/10.1080/00958964.2015.1021661

Jiménez-Aleixandre, M. P., Bugallo Rodríguez, A., \& Duschl, R. A. (2000). "Doing the lesson" or "doing science": Argument in high school genetics. Science Education, 84 (6), 757-792. https://doi.org/10.1002/1098-237X(200011)84:6<757::AID-SCE5>3.0.CO;2-F

Kandpal, T. C., \& Garg, H. P. (1999). Energy education. Applied energy, 64 (1-4), 71-78.

Kolstø S. D., \& Ratcliffe, M. (2007) Aspectos sociales de la argumentación. En: Erduran, S., Jiménez\& Aleixandre, M. P. (eds). Argumentation in Science Education. Springer, Dordrecht.

Lee, L. S., Lee, Y. F., Altschuld, J. W., \& Pan, Y. J. (2015). Energy literacy: Evaluating knowledge,affect, and behavior of students in Taiwan. Energy Policy, 76, 98-106. https://doi.org/10.1016/j.enpol.2014.11.012

Lee, L. S., Lee, Y. F., Wu, M. J., \& Pan, Y. J. (2019). Un estudio de alfabetización energética entre estudiantes de enfermería para examinar las implicaciones en los esfuerzos de conservación de energía en Taiwán. Política energética, 135, e111005

Liarakou, G., Gavrilakis, C., \& Flouri, E. (2009). Secondary school teachers' knowledge and attitudes towards renewable energy sources. Journal of Science Education and Technology, 18 (2), 120-129. https://doi.org/10.1007/s10956-008-9137-z

Martín-Gámez, C., \& Erduran, S. (2018) Comprensión de la argumentación sobre cuestiones sociocientíficas en materia de energía: un estudio cuantitativo con profesores de formación inicial en España. Investigación en educación científica y tecnológica, 36 (4), 463-483

Mirandola, A., \& Lorenzini, E. (2016). Energy, environment and climate: From the past to the future. International Journal of Heat and Technology, 34 (2), 159-164. https://doi.org/10.18280/ijht.340201

Newton, P., Driver, R., \& Jonathan, O (1999). El lugar de la argumentación en la pedagogía de la ciencia escolar. Revista Internacional de Educación en Ciencias, 21 (5), 553-576. https://doi.org/10.17227/01203916.5516

Nielsen, B. L. (2012). Science teachers' meaning-making when involved in a school-based professional development project. Journal of science teacher education, 23 (6), 621-649. https://doi.org/10.1007/s10972-012-9300-5

Ocetkiewicz, I., Tomaszewska, B., \& Mróz, A. (2017). Renewable energy in education for sustainable development. The Polish experience. Renewable and Sustainable Energy Reviews, 80, 92 97. https://doi.org/10.1016/j.rser.2017.05.144 
Pinch, T. (2015). Scientific Controversies. International Encyclopedia of the Social \& Behavioral Sciences, 21(2), 281-286. https://doi.org/10.1016/b978-0-08-097086-8.85043-6

Porter, E., \& Van Der Linde, C. (1995). Toward a new conception of the environment-competitiveness relationship. Journal of economic perspectives, $9 \quad$ (4), 97-118. https://doi.org/10.1257/jep.9.4.97

Rogoff, B., \& Chavajay, P. (2004). Las bases culturales del desarrollo cognitivo. Evolución de la investigación en este campo en Norteamérica. Revista Educación y Pedagogía, 16 (39), 121 159

Rozo-Rincón, S., Sánchez-Molina, J., \& Gelves-Díaz, J. (2014). Evaluación de minerales aluminio silicatos de Norte de Santander para fabricar piezas cerámicas de gran formato. Revista Facultad de Ingeniería, 24 (38), 53-61. https://doi.org/10.19053/01211129.3158

Sánchez-Robayo, B. J., \& Torres-Duarte, J. (2016). Aprender a investigar investigando. Realización de una propuesta de formación. Revista Científica, 1 (28), 17-31. https://doi.org/10.14483/udistrital.jour.RC.2017.28.a2

Sardà, J., \& Sanmartí, N. (2000). Enseñar a argumentar científicamente: un repte de les classes de ciències. Enseñanza de las Ciencias, 18 (3), 405-422

Sauvé, S., Bernard, S., \& Sloan, P. (2016). Environmental sciences, sustainable development and circular economy: Alternative concepts for trans-disciplinary research. Environmental Development, 17, 48-56. https://doi.org/10.1016/j.envdev.2015.09.002

Solbes-Matarredona, J., \& Torres-Merchán, N. Y. (2015). Alternativas para reflexionar aspectos críticos de la ciencia en el aula. Revista Científica, 2 (22), 31-44. https://doi.org/10.14483/10.14483/udistrital.jour.RC.2015.22.a3

Zyadin, A., Puhakka, A., Ahponen, P., Cronberg, T., \& Pelkonen, P. (2012). School students' knowledge, perceptions, and attitudes toward renewable energy in Jordan. Renewable energy, 45, 78-85. https://doi.org/10.1016/j.renene.2012.02.002 